\title{
Dipeptidyl peptidase 4 inhibitor improves brain insulin sensitivity, but fails to prevent cognitive impairment in orchiectomy obese rats
}

\author{
Hiranya Pintana ${ }^{1,2}$, Wanpitak Pongkan ${ }^{1,2}$, Wasana Pratchayasakul ${ }^{1,2}$, \\ Nipon Chattipakorn ${ }^{1,2}$ and Siriporn C Chattipakorn ${ }^{1,3}$ \\ ${ }^{1}$ Neurophysiology Unit, Faculty of Medicine, Cardiac Electrophysiology Research and Training Center, \\ ${ }^{2}$ Department of Physiology, Faculty of Medicine, and ${ }^{3}$ Department of Oral Biology and Diagnostic Science, \\ Faculty of Dentistry, Chiang Mai University, Chiang Mai 50200, Thailand
}

Correspondence should be addressed to S C Chattipakorn Email

scchattipakorn@gmail.com or siriporn.c@cmu.ac.th

\begin{abstract}
It is unclear whether the dipeptidyl peptidase 4 (DPP4) inhibitor can counteract brain insulin resistance, brain mitochondrial dysfunction, impairment of hippocampal synaptic plasticity and cognitive decline in testosterone-deprived obese rats. We hypothesized that DPP4 inhibitor vildagliptin improves cognitive function in testosterone-deprived obese rats by restoring brain insulin sensitivity, brain mitochondrial function and hippocampal synaptic plasticity. Thirty male Wistar rats received either a sham-operated $(S, n=6)$ or bilateral orchiectomy (ORX, $n=24)$. ORX rats were divided into two groups and fed with either a normal diet (ND (NDO)) or a high-fat diet (HFO) for 12 weeks. Then, ORX rats in each dietary group were divided into two subgroups ( $n=6 /$ subgroup) to receive either a vehicle or vildagliptin ( $3 \mathrm{mg} / \mathrm{kg}$ per day, p.o.) for 4 weeks. After treatment, cognitive function, metabolic parameters, brain insulin sensitivity, hippocampal synaptic plasticity and brain mitochondrial function were determined in each rat. We found that HFO rats exhibited peripheral and brain insulin resistance, brain mitochondrial dysfunction, impaired hippocampal synaptic plasticity and cognitive decline. NDO rats did not develop peripheral and brain insulin resistance. However, impaired hippocampal synaptic plasticity and cognitive decline occurred. Vildagliptin significantly improved peripheral insulin sensitivity, restored brain insulin sensitivity and decreased brain mitochondrial reactive oxygen species production in HFO rats. However, vildagliptin did not restore hippocampal synaptic plasticity and cognitive function in both NDO and HFO rats. These findings suggest that vildagliptin could not counteract the impairment of hippocampal synaptic plasticity and cognitive decline in testosterone-deprived subjects, despite its effects on improved peripheral and brain insulin sensitivity as well as brain mitochondrial function.
\end{abstract}

\section{Key Words}

- vildagliptin

- obese-insulin resistance

- testosterone deprivation

- brain mitochondrial function

- hippocampal synaptic plasticity

- cognitive function Printed in Great Britain
Published by Bioscientifica Ltd
Journal of Endocrinology (2015) 226, M1-M11 


\section{Introduction}

Long-term high-calorie diets cause obesity, leading to the development of insulin resistance, metabolic syndrome and type 2 diabetes mellitus (Pandolfi et al. 1994, Kahn \& Flier 2000). Moreover, several studies demonstrated that insulin resistance was also associated with cognitive decline (Craft 2005, Greenwood \& Winocur 2005, Stranahan et al. 2008). Our studies also showed that 12weeks of a high-fat diet (HFD) consumption in male rats induced not only obese-insulin resistance, but also impaired brain insulin sensitivity, brain mitochondrial dysfunction and cognitive decline (Pintana et al. 2012, 2013, Pipatpiboon et al. 2013).

Testosterone is an androgenic steroid hormone, which plays an important role in the regulation of male reproduction and behaviors, as well as in the maintenance of glucose and lipid metabolism (Aydilek \& Aksakal 2005, Christoffersen et al. 2010). Several studies have shown that testosterone replacement exerted beneficial effects on brain function, including the prevention of neuronal cell death, the balancing of brain oxidative stress and antioxidant activity, improvement of synaptic plasticity and the involvement of cognitive formation in animal models (Janowsky 2006, Meydan et al. 2010, Filova et al. 2013). Interestingly, growing evidence demonstrated that testosterone deficiency is associated with obese insulinresistance in men (Haffner et al. 1994, Stellato et al. 2000, Grossmann et al. 2008, Shi et al. 2013). All of those findings indicate that testosterone-deprivation can lead to obesity and cognitive decline. Therefore, it is possible that testosterone deprivation combined with obesity could lead to the worsening of cognitive decline.

The dipeptidyl peptidase 4 (DPP4) inhibitor, vildagliptin, is an anti-diabetic drug, which exerts beneficial effects in diabetic and insulin resistant models, such as the regulation of blood glucose levels without hypoglycemic incidence (Burkey et al. 2005), restoring pancreatic $\beta$-cells as well as preserving pancreatic function and improving insulin sensitivity (Ahren et al. 2004). Our previous study demonstrated that vildagliptin improved not only peripheral insulin sensitivity, but also increased brain insulin sensitivity in obese-insulin resistant rats, as indicated by improvement in brain insulin receptor (IR) function (Pipatpiboon et al. 2013). In addition, vildagliptin have been shown to enhance hippocampal neurogenesis, leading to improved learning and memory in HFD-fed rodents (Pintana et al. 2013, Pipatpiboon et al. 2013, Gault et al. 2015). All of these findings indicate the beneficial effects of vildagliptin on peripheral insulin sensitivity, brain insulin signaling and cognitive function in obeseinsulin resistant conditions. However, the effects of vildagliptin on brain insulin sensitivity, brain mitochondrial function, hippocampal synaptic plasticity and cognitive function in testosterone-deprived obese rats have never been investigated. We tested the hypothesis that testosterone-deprived rats with or without obesity have impaired brain IR function, decreased brain insulin signaling, impaired brain mitochondrial function and impaired hippocampal synaptic plasticity, thus leading to cognitive decline. We also hypothesized that vildagliptin could restore these impairments in testosterone-deprived conditions with or without obesity.

\section{Materials and methods}

\section{Animal models and experimental protocols}

Thirty male Wistar rats, weighing 180-200 g (aged 5-6 weeks old) obtained from the National Animal Center, Salaya Campus, Mahidol University, Bangkok, Thailand were used. All experiments were conducted in accordance with the approved protocol from the Faculty of Medicine, Chiang Mai University Institutional Animal Care and Use Committee, in compliance with NIH guidelines. All animals were housed in environmentally controlled conditions $\left(25 \pm 0.5^{\circ} \mathrm{C}\right.$ and a $12 \mathrm{~h}$ light: $12 \mathrm{~h}$ darkness cycle) and allowed to acclimate for 1 week. Rats were then divided into two groups as either control sham-operated (S, $n=6$ ) or bilateral orchiectomized (ORX, $n=24$ ) rats as described previously (Dulisch 1976). Rats in the shamoperated group were fed with a ND for 12 weeks and were the control group (NDS); after that rats received vehicle until the end of experiment. Rats in ORX group were randomized and fed with either a normal diet (ND: $19.77 \% \mathrm{E}$ fat, NDO) or a HFD (59.28\% E fat, HFO) for 12 weeks (Pratchayasakul et al. 2011). At the end of 12 weeks, the rats in each group were divided into two subgroups ( $n=6 /$ subgroup) and received either the vehicle (normal saline solution; $2 \mathrm{ml} / \mathrm{kg}$ per day) (NDO rats treated with vehicle (NDOV) and HFO rats treated with vehicle (HFOV)) or vildagliptin (Gulvus, Novartis, Bangkok, Thailand; $3 \mathrm{mg} / \mathrm{kg}$ per day) (NDO rats treated with vildagliptin (NDOVil) and HFO rats treated with vildagliptin (HFOVil)), via gavage feeding for 4 weeks (Burkey et al. 2005, Pintana et al. 2013). The cognitive function was subsequently determined by the Morris water maze (MWM) test. Blood samples were collected from a tail

Published by Bioscientifica Ltd 
vein at week 12, and week 15 for further plasma analysis. At the end of the experimental period, rats were deeply anesthetized with isoflurane and killed by decapitation. The brain of each rat was removed as quickly as possible after death and carefully sliced in preparation for extracellular recording (insulin-induced long-term depression (LTD) and hippocampal synaptic long-term potentiation (LTP)), immunoblot and brain mitochondrial functions. The study protocol is shown in Fig. 1.

\section{Blood sample assays}

Plasma glucose and cholesterol levels were determined via colorimetric assay (Biotech, Bangkok, Thailand). Plasma HDL and LDL levels were determined using a commercial colorimetric assay kit (Biovision, CA, USA). Plasma insulin levels were determined using the Sandwich ELISA Kit (Linco Research, St Charles, MO, USA). Peripheral insulin resistance was assessed using the homeostasis model assessment (HOMA), which was calculated by the following formula: (fasting plasma insulin $(\mu \mathrm{U} / \mathrm{ml})) \times$ (fasting plasma glucose $(\mathrm{mmol} / \mathrm{l})) / 22.5$ as previously described (Pipatpiboon et al. 2012). Plasma testosterone levels were measured using the electrochemiluminescence immunoassay (ECLIA) technique (Roche Diagnostics).

\section{Oral glucose tolerance test}

Oral glucose tolerance test (OGTT) was performed as described previously (Pipatpiboon et al. 2013). Briefly, rats were starved overnight before the test and received $2 \mathrm{~g} / \mathrm{kg}$ glucose solution via oral gavage. Blood samples were collected from the tail vein at 0, 15, 30, 60, 90 and $120 \mathrm{~min}$ after glucose administration. Areas under the curves (AUC) were calculated to evaluate glucose tolerance.

\section{Brain slice preparation}

At the end of experiment, animals were anesthetized with isoflurane and decapitated. The brain slice preparation was

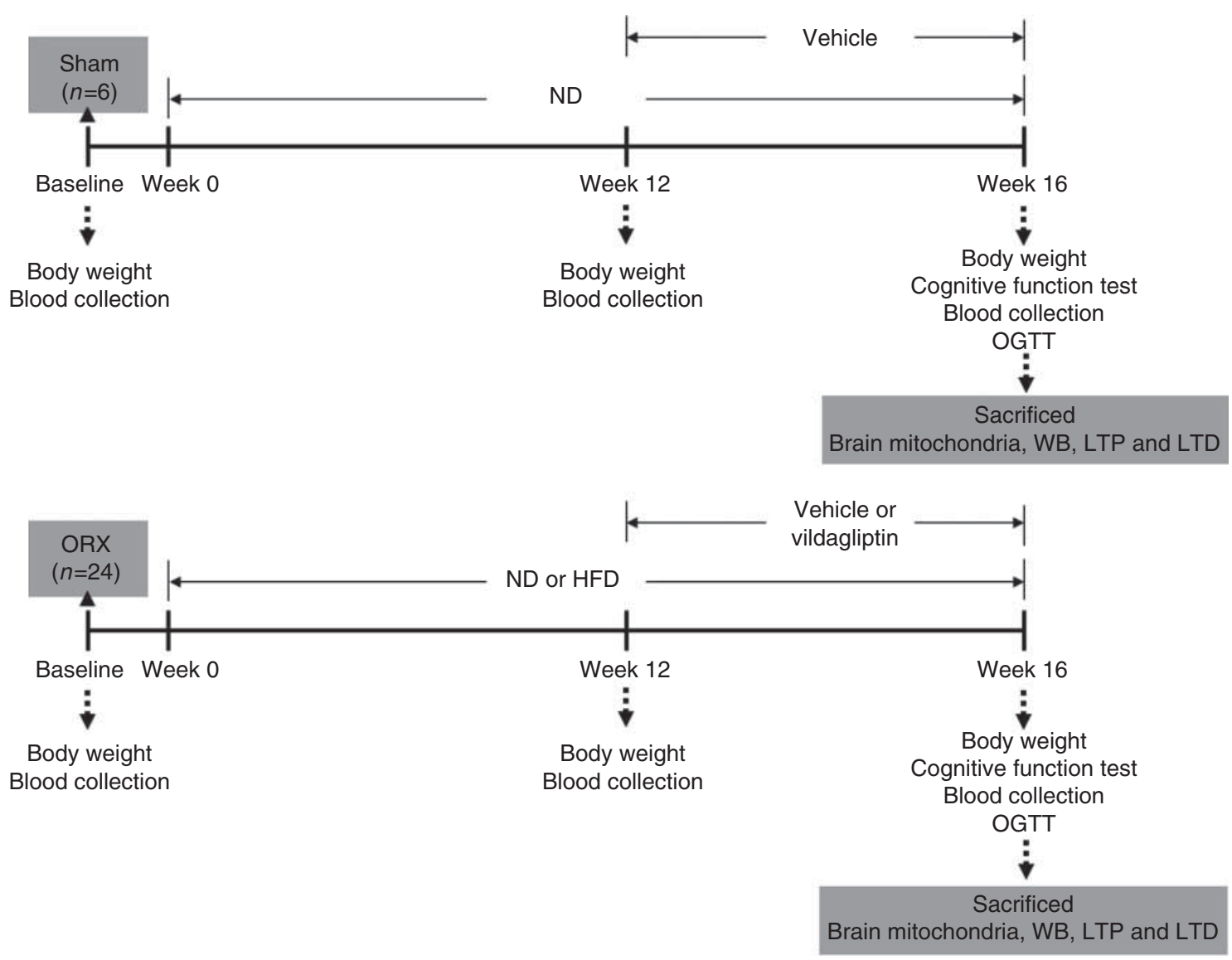

Figure 1

The experimental protocol of the present study. 
performed as described previously (Pratchayasakul et al. 2011, Pipatpiboon et al. 2012, 2013).

\section{Extracellular recordings of hippocampal slices for insulin-induced LTD}

An extracellular recording of hippocampal slices for insulin-induced LTD was performed as described in our previous studies (Pratchayasakul et al. 2011, Pipatpiboon et al. 2012, 2013). Field excitatory postsynaptic potentials (fEPSPs) were evoked by stimulating the Schaffer collateralcommissural pathway with a bipolar tungsten electrode, while recordings were taken from the stratum radiatum of the hippocampal CA1 region with micropipettes (3 Mohm) filled with $2 \mathrm{M} \mathrm{NaCl}$. Stimulus frequency was $0.033 \mathrm{~Hz}$. The stimulus intensity of fEPSPs was adjusted to $0.8-1.0 \mathrm{mV}$ in amplitude. Hippocampal slices were perfused with aCSF (as baseline condition) for $10 \mathrm{~min}$, and then perfused with aCSF plus $500 \mathrm{nM}$ insulin (as insulin-induced LTD) for an additional $10 \mathrm{~min}$. Afterward, the slices were perfused with aCSF for an additional $50 \mathrm{~min}$ and readings recorded. Data was filtered at $3 \mathrm{kHz}$, digitized at $10 \mathrm{kHz}$, and stored in a computer using pClamp 9.2 Software (Axon Instruments, Union City, CA, USA). The initial slope of the fEPSP was measured and plotted against time.

\section{Extracellular recordings of hippocampal slices for synaptic LTP}

The examination of electrical-induced hippocampal synaptic LTP was performed as described in our previous study (Sripetchwandee et al. 2014). fEPSPs were evoked by stimulating the Schaffer collateral-commissural pathway with a bipolar tungsten electrode, while the fEPSPs recordings were taken from the stratum radiatum of the hippocampal CA1 region with micropipettes (3 Mohm) filled with $2 \mathrm{M} \mathrm{NaCl}$. LTP was induced by delivering highfrequency stimulation (HFS; four trains at $100 \mathrm{~Hz} ; 0.5 \mathrm{~s}$ duration; $20 \mathrm{~s}$ interval). Experiments were performed for at least $40 \mathrm{~min}$ after HFS. The amount of potentiation was calculated at $40 \mathrm{~min}$ after tetanus. All data were filtered at $3 \mathrm{kHz}$, digitized at $10 \mathrm{kHz}$, and stored in a computer using pClamp9.2 Software (Axon Instruments). The initial slopes of the fEPSPs were measured and plotted against time.

\section{Immunoblotting for brain insulin signaling}

To investigate the expression of IR phosphorylation (p-IR) and IRs, homogenate brain slices from each subgroup were used as described in our previous study (Pratchayasakul et al. 2011, Pipatpiboon et al. 2012, 2013). IR tyrosine phosphorylation were electrophoresed and immunoblotted with rabbit antibodies for IR at tyrosine respectively. Examination of the level of IR protein expression was conducted with homogenates prepared from another set of four whole brain slices. These proteins were resolved by an immunoblot assay conducted with rabbit anti-IR at tyrosine phosphorylation ( $\mathrm{p}-\mathrm{IR}^{\mathrm{Ty} 1162 / 1163} ; 1: 1000$; sc-25103-R; Santa Cruz Biotechnology, Dallas, TX, USA), IR (1:1000; sc-711; Santa Cruz Biotechnology). For a loading control, immunoblotting for each membrane was incubated with anti- $\beta$-actin (1:4000; \#4967; Cell Signaling Technology, Danvers, MA, USA). All membranes for visualizing the phosphorylation and the protein levels of IR expression were incubated with secondary goat antirabbit antibody conjugated with HRP (1:2000; \#7074; Cell Signaling Technology). The protein bands were visualized on Amersham Hyperfilm ECL (GE Healthcare, Buckinghamshire, UK) using Amersham ECL Western Blot Detection Reagents (GE Healthcare). Band densities of phosphorylated IR were represented as a ratio of insulin stimulation $(+)$ to no insulin stimulation $(-)$ and were normalized to total IR. In addition, band intensities were quantified by Scion Image (Scion Corporation, National Institute of Health, Federick, MD, USA) and the results were shown as average signal intensity (arbitrary) units.

\section{Preparation of brain mitochondria}

Brain mitochondria were isolated using the method described previously (Pipatpiboon et al. 2013). Mitochondrial protein concentration was measured using BCA assay (Pipatpiboon et al. 2013). Brain mitochondrial functions including brain mitochondrial reactive oxygen species (ROS), mitochondrial membrane potential change $(\Delta \Psi \mathrm{m})$ and mitochondrial swelling were also determined.

Brain mitochondrial ROS assay Brain mitochondrial ROS were measured using dichloro-hydrofluoresceindiacetate (DCFHDA) fluorescent dye. Brain mitochondria $(0.4 \mathrm{mg} / \mathrm{ml})$ were incubated with $2-\mu \mathrm{M}$ DCFHDA at $25^{\circ} \mathrm{C}$ for $20 \mathrm{~min}$. The fluorescence was determined using a fluorescent microplate reader at the excitation wavelength of $485 \mathrm{~nm}$ and emission wavelength of $530 \mathrm{~nm}$ (Pipatpiboon et al. 2013). Increased fluorescence intensity indicates increased ROS production.

Brain $\Delta \Psi \mathrm{m}$ assay The change in $\Delta \Psi \mathrm{m}$ was measured using the fluorescent dye 5, $5^{\prime}, 6,6^{\prime}$-tetrachloro-1, $1^{\prime}, 3,3^{\prime}$ tetraethyl benzimidazolcarbocyanine iodide (JC-1).

Published by Bioscientifica Ltd. 
The JC-1 monomer form (green fluorescence) was excited at a wavelength of $485 \mathrm{~nm}$ and detected at the emission wavelength of $590 \mathrm{~nm}$. JC-1 aggregate form (red fluorescence) was excited at a wavelength of $485 \mathrm{~nm}$ and detected at the emission wavelength of $530 \mathrm{~nm}$. Brain mitochondria $(0.4 \mathrm{mg} / \mathrm{ml})$ were incubated with JC-1 dye at $37^{\circ} \mathrm{C}$ for $15 \mathrm{~min} . \Delta \Psi \mathrm{m}$ changes were determined by measuring the fluorescence intensity using a fluorescent microplate reader. The change in $\Delta \Psi \mathrm{m}$ was calculated as the ratio of red to green fluorescent intensity (Pipatpiboon et al. 2013). Decreased red/green fluorescence intensity ratio indicates mitochondrial depolarization.

Brain mitochondrial swelling assay Brain mitochondrial swelling was determined by measuring the change in the absorbance of brain mitochondrial suspension. Brain mitochondria $(0.4 \mathrm{mg} / \mathrm{ml})$ were incubated in $2 \mathrm{ml}$ of respiration buffer. The suspension was read at $540 \mathrm{~nm}$ using a microplate reader. Mitochondrial swelling was indicated by a decrease in the absorbance (Pipatpiboon et al. 2013).

\section{Cognitive function test}

The open-field test (OFT) was used to screen locomotor activity via counting the number of lines that the rats crossed during the test, as described in previous studies (Arakawa 2005, Pintana et al. 2013). The assessment of cognitive function was performed by using MWM with two assessments including the acquisition test which was carried out for 5 consecutive days, and the probe test which was performed on day 6 (Vorhees \& Williams 2006, Pintana et al. 2013). Data analysis of MWM test was done manually from video tape recordings by the investigators, who were blinded to experimental groups.

\section{Statistical analysis}

Data were expressed as mean \pm s.E.M. For all comparisons, the significance of the differences in peripheral biochemical parameters was calculated using the MannWhitney $U$ test. The comparisons in the percentage of insulin-induced LTD, the percentage of LTP, brain mitochondrial function, immunoblot, the OFTs and the MWM tests for the probe test among groups were performed using the one-way ANOVA test, followed by post-hoc Fisher's least significant difference (LSD) analysis. Comparisons among groups in MWM tests for the acquisition test were performed using a two-way ANOVA, followed by post-hoc LSD analysis. $P<0.05$ was considered statistically significant.

\section{Results}

Obesity, but not testosterone deprivation, caused peripheral insulin resistance, which was attenuated by vildagliptin treatment

At the baseline levels (before ORX), the metabolic parameters were not significantly different between the sham-operated group and the ORX group (Table 1). After 12 weeks of diet regimens, HFO rats had significantly increased plasma insulin, HOMA index and total cholesterol levels, when compared with NDS and NDO rats. Plasma glucose levels were not significantly different among groups. However, the body weight of NDO had significantly decreased, when compared with NDS (Table 1). These findings suggested that long-term HFD consumption caused peripheral insulin resistance as indicated by hyperinsulinemia with euglycemia and increased HOMA index. However, testosterone deprivation alone did not impair peripheral insulin sensitivity.

After 4 weeks of vildagliptin treatment, testosterone deprivation by ORX was confirmed by reduced plasma testosterone levels $(<0.025 \mathrm{ng} / \mathrm{ml})$ in all ORX groups (NDOV, NDOVil, HFOV and HFOVil) compared to NDS group $(0.75 \pm 0.06 \mathrm{ng} / \mathrm{ml})$. NDOV and NDOVil had significantly decreased body weight and visceral fat, without any significant alteration in plasma insulin, HOMA index,

Table 1 Metabolic parameters at baseline and the end of week 12 (before treatment)

\begin{tabular}{|c|c|c|}
\hline \multirow[b]{2}{*}{ Metabolic parameters } & \multicolumn{2}{|c|}{ Baseline } \\
\hline & Sham & ORX \\
\hline Body weight (g) & $192.5 \pm 3.7$ & $197.5 \pm 3.7$ \\
\hline Plasma glucose (mg/dl) & $106.8 \pm 4.5$ & $111.8 \pm 4.3$ \\
\hline Plasma insulin (ng/ml) & $2.1 \pm 0.3$ & $2.2 \pm 0.4$ \\
\hline HOMA index & $13.4 \pm 1.2$ & $14.0 \pm 2.2$ \\
\hline Plasma total cholesterol (mg/dl) & $45.5 \pm 2.2$ & $47.7 \pm 1.7$ \\
\hline
\end{tabular}

\begin{tabular}{|c|c|c|}
\hline \multicolumn{3}{|c|}{ Week 12} \\
\hline NDS & NDO & HFO \\
\hline $466.6 \pm 4.9$ & $409.2 \pm 14.1^{a, b}$ & $472.0 \pm 8.4$ \\
\hline $133.8 \pm 4.9$ & $140.0 \pm 5.5$ & $139.9 \pm 7.1$ \\
\hline $2.0 \pm 0.2$ & $2.1 \pm 0.2^{b}$ & $3.7 \pm 0.3^{a}$ \\
\hline $15.3 \pm 2.0$ & $17.7 \pm 2.1^{b}$ & $27.6 \pm 3.4^{a}$ \\
\hline $48.5 \pm 2.7$ & $52.1 \pm 2.0^{b}$ & $75.0 \pm 6.6^{a}$ \\
\hline
\end{tabular}

${ }^{b}$ Compared to HFO. 
Table 2 Metabolic parameters after vildagliptin treatment for 4 weeks

\begin{tabular}{|c|c|c|c|c|c|}
\hline \multirow[b]{2}{*}{ Metabolic parameters } & \multicolumn{5}{|c|}{ Groups } \\
\hline & NDS & NDOV & NDOVil & HFOV & HFOVil \\
\hline Body weight (g) & $496.5 \pm 10.7$ & $423.3 \pm 8.4^{a, b}$ & $417.2 \pm 17.6^{\mathrm{a}, \mathrm{b}}$ & $506.6 \pm 17.8$ & $496.6 \pm 16.4$ \\
\hline Visceral fat $(g)$ & $26.8 \pm 1.6$ & $16.0 \pm 1.9^{\mathrm{a}, \mathrm{b}}$ & $15.7 \pm 1.4^{\mathrm{a}, \mathrm{b}}$ & $31.3 \pm 3.4$ & $30.3 \pm 3.3$ \\
\hline Plasma glucose (mg/dl) & $131.5 \pm 10.6$ & $129.0 \pm 13.1$ & $137.3 \pm 3.1$ & $134.1 \pm 8.9$ & $131.7 \pm 6.3$ \\
\hline Plasma insulin $(\mathrm{ng} / \mathrm{ml})$ & $2.53 \pm 0.34$ & $2.78 \pm 0.18$ & $2.71 \pm 0.36$ & $5.07 \pm 1.16^{\mathrm{a}}$ & $2.37 \pm 0.37^{b}$ \\
\hline HOMA index & $21.3 \pm 2.9$ & $24.4 \pm 2.8$ & $21.5 \pm 3.4$ & $44.7 \pm 13.8^{a}$ & $20.6 \pm 3.8^{\mathrm{b}}$ \\
\hline $\begin{array}{l}\text { Plasma glucose AUC (AUCg) } \\
\left(\mathrm{mg} / \mathrm{dl} \times \min \times 10^{4}\right)\end{array}$ & $4.47 \pm 0.20$ & $5.01 \pm 0.33$ & $4.48 \pm 0.23$ & $5.92 \pm 0.25^{\mathrm{a}}$ & $4.97 \pm 0.26^{\mathrm{b}}$ \\
\hline Plasma total cholesterol (mg/dl) & $68.0 \pm 5.5$ & $63.8 \pm 2.6$ & $73.3 \pm 5.8$ & $110.8 \pm 14.9^{a}$ & $101.7 \pm 8.7^{a}$ \\
\hline Plasma triglyceride $(\mathrm{mg} / \mathrm{dl})$ & $51.9 \pm 2.2$ & $55.3 \pm 9.9$ & $57.4 \pm 6.1$ & $49.8 \pm 4.1$ & $50.2 \pm 7.5$ \\
\hline HDL cholesterol (mg/dl) & $6.7 \pm 0.4$ & $7.2 \pm 0.3$ & $7.5 \pm 0.4$ & $4.9 \pm 0.6^{\mathrm{a}}$ & $6.9 \pm 0.4^{b}$ \\
\hline LDL cholesterol (mg/dl) & $60.6 \pm 7.5$ & $52.9 \pm 3.6$ & $56.9 \pm 4.0$ & $116.4 \pm 15.7^{a}$ & $81.5 \pm 8.1^{b}$ \\
\hline
\end{tabular}

plasma glucose AUC of OGTT (AUCg), total cholesterol, plasma LDL cholesterol levels as well as plasma HDL cholesterol levels, when compared with those of NDS rats (Table 2). These findings indicated that peripheral insulin resistance was not observed in the NDOV and NDOVil rats. In contrast, HFOV showed the characteristics of peripheral insulin resistance, including increased plasma insulin, HOMA index, plasma AUCg, total cholesterol, plasma LDL cholesterol levels as well as decreased plasma HDL cholesterol levels, when compared with those of NDS rats. However, plasma glucose and plasma triglyceride levels showed no significant difference among all groups. Interestingly, HFOVil had significantly decreased plasma insulin, HOMA index, plasma AUCg and plasma LDL cholesterol levels as well as increased plasma HDL cholesterol levels, when compared with those of HFOV rats. All of these findings suggested that vildagliptin attenuated peripheral insulin resistance via increased peripheral insulin sensitivity and improved lipid profiles in testosterone-deprived obese rats.

\section{Obesity, but not testosterone deprivation, caused the impairment of brain IR function, which was attenuated by vildagliptin treatment}

Brain IR function was tested by measuring insulin-induced LTD and brain insulin signaling. For the insulin-induced LTD study, the degrees of insulin-mediated LTD in ND-fed rats (NDS, NDOV and NDOVil) were not significantly different among groups $(n=2-3$ independent slices/ animal, $n=6$ animals/group, Fig. 2A). However, the degree of insulin-induced LTD was significantly reduced in HFOV rats, when compared with that of NDS rats $(n=2-3$ independent slices/animal, $n=6$ animals/group, Fig. 2A).
After 4 weeks of vildagliptin administration, the degrees of insulin-induced LTD in HFOVil was significantly increased compared with that of HFOV rats $(n=2-3$ independent slices/animal, $n=6$ animals/group, Fig. 2A).

For the brain insulin signaling study, the expression of IRs in all groups showed no significant different among them (Fig. 2C). The p-IRs at tyrosine site was not significantly different among groups of ND-fed rats (NDS, NDOV and NDOVil; Fig. 2B). However, levels of p-IR of HFOV rats were significantly decreased, when compared with those of NDOV rats (Fig. 2B). Furthermore, vildagliptin treatment significantly increased p-IR levels in HFO rats (Fig. 2B). All of these findings suggested that the obese insulin-resistant condition, but not testosterone deprivation, caused the impairment of brain IR function and brain insulin signaling. In addition, vildagliptin restored the impairment of brain IR function as well as brain insulin signaling in the testosterone-deprived obese condition.

Obesity, but not testosterone deprivation, caused brain mitochondrial dysfunction and vildagliptin attenuated that impairment

Brain mitochondrial function was determined by assessing changes in brain mitochondrial ROS production and brain $\Delta \Psi \mathrm{m}$ change after $\mathrm{H}_{2} \mathrm{O}_{2}$ stimulation, and brain mitochondrial swelling. The results demonstrated that brain mitochondrial function, including brain mitochondrial ROS level, brain $\Delta \Psi \mathrm{m}$ changes and brain mitochondrial swelling, was not significantly different among groups of ND-fed rats (NDS, NDOV and NDOVil; Fig. 3A, B and C). These findings suggested that testosterone deprivation did not impair brain mitochondrial function. Furthermore, HFOV rats had a significant increase in brain

Published by Bioscientifica Ltd 
A
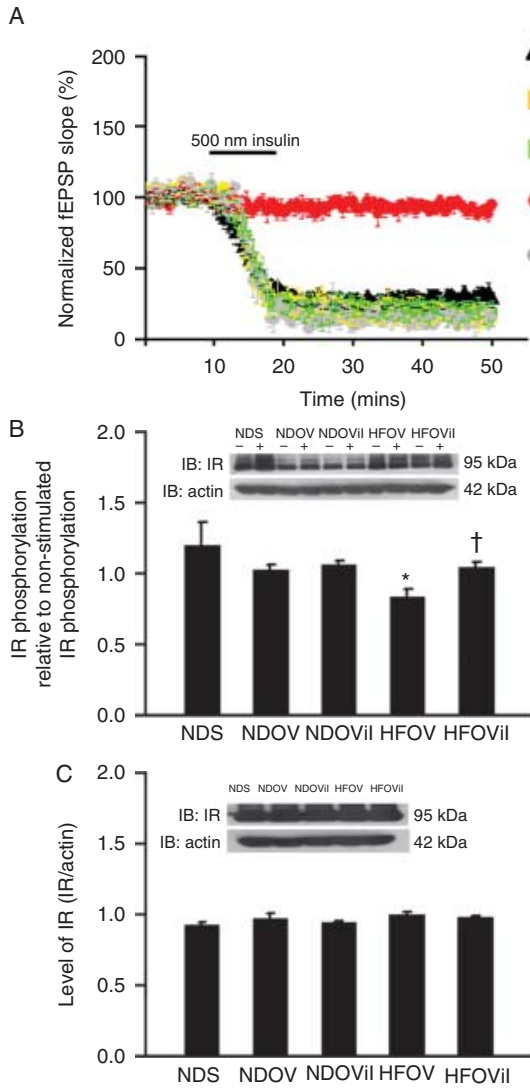

Figure 2

The effects of vildagliptin on brain insulin receptor (IR) function, including insulin-induced LTD, the phosphorylation of IR $(p-I R)$ and the expression of IRs in testosterone-deprived rats with or without obesity. Insulin-induced LTD in the CA1 hippocampus and the p-IR significantly decreased in HFOV rats, when compared with that of NDS rats ( $A$ and $B$ ). The administration of vildagliptin for 4 weeks significantly improved the ability of insulininduced LTD and the $p$-IR in HFOVil rats, when compared with that of HFOV rats ( $A$ and $B$ ). No differences in the expression of $I R$ among all groups were found (C). NDS, sham-operated rats with normal diet feeding; NDOV, orchiectomized-operated rats with normal diet feeding and treated with vehicle; NDOVil, orchiectomized-operated rats with normal diet feeding and treated with vildagliptin; HFOV, orchiectomized-operated rats with high-fat diet feeding and treated with vehicle; HFOVil, orchiectomizedoperated rats fed with a high-fat diet and treated with vildagliptin. $* P<0.05$ vs NDS and ${ }^{\dagger} P<0.05$ vs HFOV. A full colour version of this figure is available at http://dx.doi.org/10.1530/JOE-15-0099.

mitochondrial ROS production, brain mitochondrial depolarization and brain mitochondrial swelling, when compared with those of NDOV rats (Fig. 3A, B and C). In addition, vildagliptin treatment significantly decreased brain ROS production in HFOVil rats, when compared with that of HFOV rats (Fig. 3A). However, vildagliptin did not attenuate brain mitochondrial depolarization and brain mitochondrial swelling in HFO rats (Fig. 3B and C). The morphological changes of brain mitochondria from all groups were shown in Fig. 4. Intact brain mitochondrial morphology with apparent folded cristae was observed in all ND-fed rats (Fig. 4A, B and C). Brain mitochondrial swelling was observed in both the HFOV and HFOVil groups as indicated by markedly unfolded cristae (Fig. 4D and E). All of these findings suggested that the obese
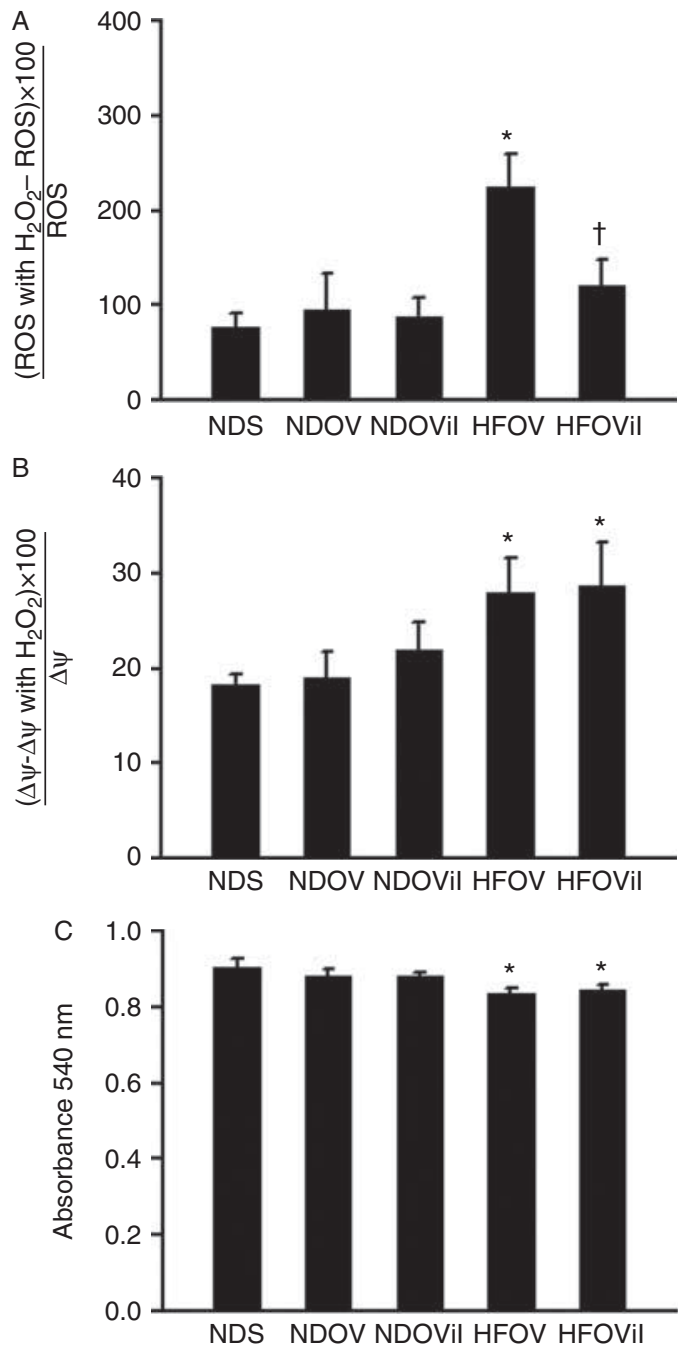

Figure 3

The effects of vildagliptin on brain mitochondrial function in testosterone deprived rats with or without obesity. HFOV rats demonstrated brain mitochondrial dysfunction, as indicated by increased brain mitochondrial ROS production following $\mathrm{H}_{2} \mathrm{O}_{2}$ application (A), increased brain mitochondrial membrane potential $(\Delta \Psi \mathrm{m})$ change following $\mathrm{H}_{2} \mathrm{O}_{2}$ application (B) and decreased absorbance values, indicating brain mitochondrial swelling (C). The administration of vildagliptin for 4 weeks significantly decreased brain mitochondrial ROS production in HFOVil rats, when compared with that of HFOV rats (A). However, vildagliptin did not improve brain $\triangle \Psi m$ change and brain mitochondrial swelling in HFOVil rats (B and C). NDS, sham-operated rats with normal diet feeding; NDOV, orchiectomized-operated rats with normal diet feeding and treated with vehicle; NDOVil, orchiectomized-operated rats with normal diet feeding and treated with vildagliptin; HFOV, orchiectomized-operated rats with high-fat diet feeding and treated with vehicle; HFOVil, orchiectomizedoperated rats with high-fat diet feeding and treated with vildagliptin. ${ }^{*} P<0.05$ vs NDOV and ${ }^{\dagger} P<0.05$ vs HFOV.

Published by Bioscientifica Ltd 

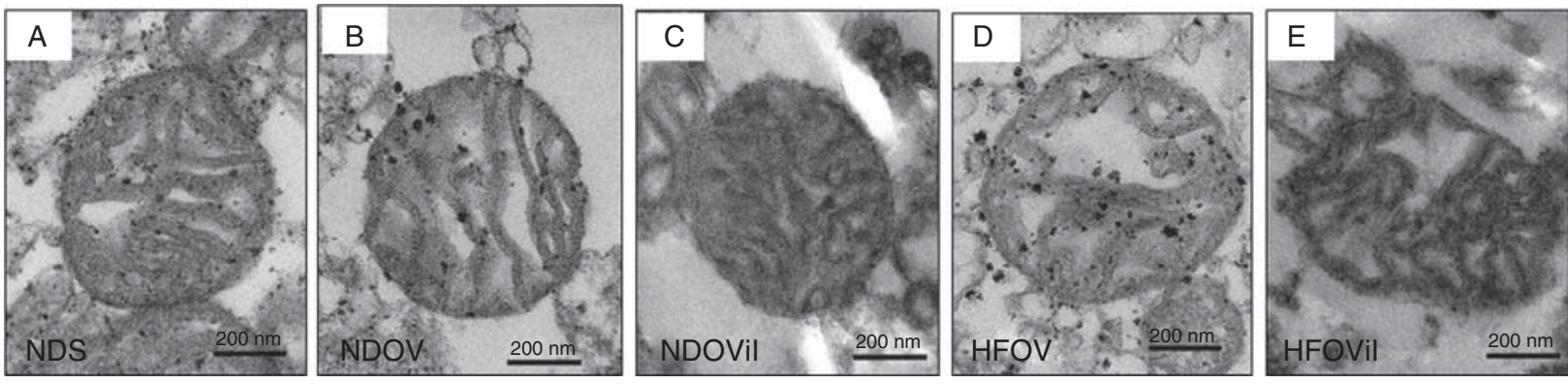

Figure 4

Representative images of brain mitochondrial morphology by transmission electron microscopy (JEM-2200FS field emission electron microscope, original magnification $\times 20000$ ) after 4 weeks of vildagliptin treatment. Normal folding of cristae in brain mitochondrial morphology was shown among all normal diet-fed rats (A, B and C). However, brain mitochondrial swelling, as indicated by unfolded cristae, of both HFOV and HFOVil groups was observed ( $D$ and $E$ ). NDS, sham-operated rats with normal diet feeding;

insulin-resistant condition, but not testosterone deprivation, caused the impairment of brain mitochondrial function. In addition, vildagliptin attenuated brain mitochondrial dysfunction by reducing brain ROS production in the testosterone-deprived obese condition.

\section{Testosterone deprivation with or without obesity caused impaired hippocampal synaptic plasticity and cognitive decline, and vildagliptin failed to restore those impairments}

To study hippocampal synaptic plasticity, electricalinduced LTP was determined. The results demonstrated that the degrees of electrical-induced LTP significantly decreased in both NDOV and HFOV rats, when compared with that of NDS rats. The reduction of electrical-induced LTP in both NDOV and HFOV rats $(n=2-3$ independent slices/animal, $n=6$ animals/group, Fig. 5A) was not significantly different. In addition, vildagliptin administration did not improve the degree of electrical-induced LTP in both NDO and HFO rats. These findings indicated that testosterone deprivation with or without obesity caused the impairment of hippocampal synaptic plasticity. However, vildagliptin treatment could not attenuate that impairment.

Furthermore, the OFT was used to screen locomotor activity. The results showed that it was not significantly different among all groups, indicating that the locomotor activity did not differ in all groups. In addition, the present study determined cognitive function by using the MWM test. For the acquisition test, the time to reach the platform was not significantly different among ND-fed rats (NDS, NDOV and NDOVil rats; Fig. 5B). However, we
NDOV, orchiectomized-operated rats with normal diet feeding and treated with vehicle; NDOVil, orchiectomized-operated rats with normal diet feeding and treated with vildagliptin; HFOV, orchiectomized-operated rats with high-fat diet feeding and treated with vehicle; HFOVil, orchiectomized-operated rats with high-fat diet feeding and treated with vildagliptin.

found that HFOV and HFOVil rats took a longer time to reach the platform, when compared with that of NDS rats (Fig. 5B). For the probe test, the results showed that time spent in the target quadrant of the NDOV, NDOVil, HFOV and HFOVil rats was significantly decreased compared to NDS rats (Fig. 5C). These findings suggested that testosterone deprivation with or without obesity caused cognitive decline, and vildagliptin failed to restore cognitive function in the testosterone-deprived rats.

\section{Discussion}

The major findings of the present study are as follows: i) the condition of obesity in testosterone-deprived rats leads to the development of peripheral insulin resistance, impairment of brain insulin sensitivity, brain mitochondrial dysfunction and impaired hippocampal synaptic plasticity, resulting in cognitive decline; ii) vildagliptin improves peripheral insulin sensitivity, brain insulin sensitivity and brain mitochondrial function in testosterone-deprived obese rats; and iii) vildagliptin fails to restore hippocampal synaptic plasticity and cognitive function in testosterone deprived rats.

The present study demonstrates that peripheral insulin resistance, as indicated by increased HOMA index and impaired OGTT as well as impaired lipid profiles, developed in testosterone-deprived rats with obesity. Consistent with these findings, previous studies demonstrated that HFD-induced obesity caused the development of peripheral insulin resistance in male rats (Akiyama et al. 1996, Stranahan et al. 2008, Pintana et al. 2013, Pipatpiboon et al. 2013). The development of peripheral insulin resistance in the present study

Published by Bioscientifica Ltd 

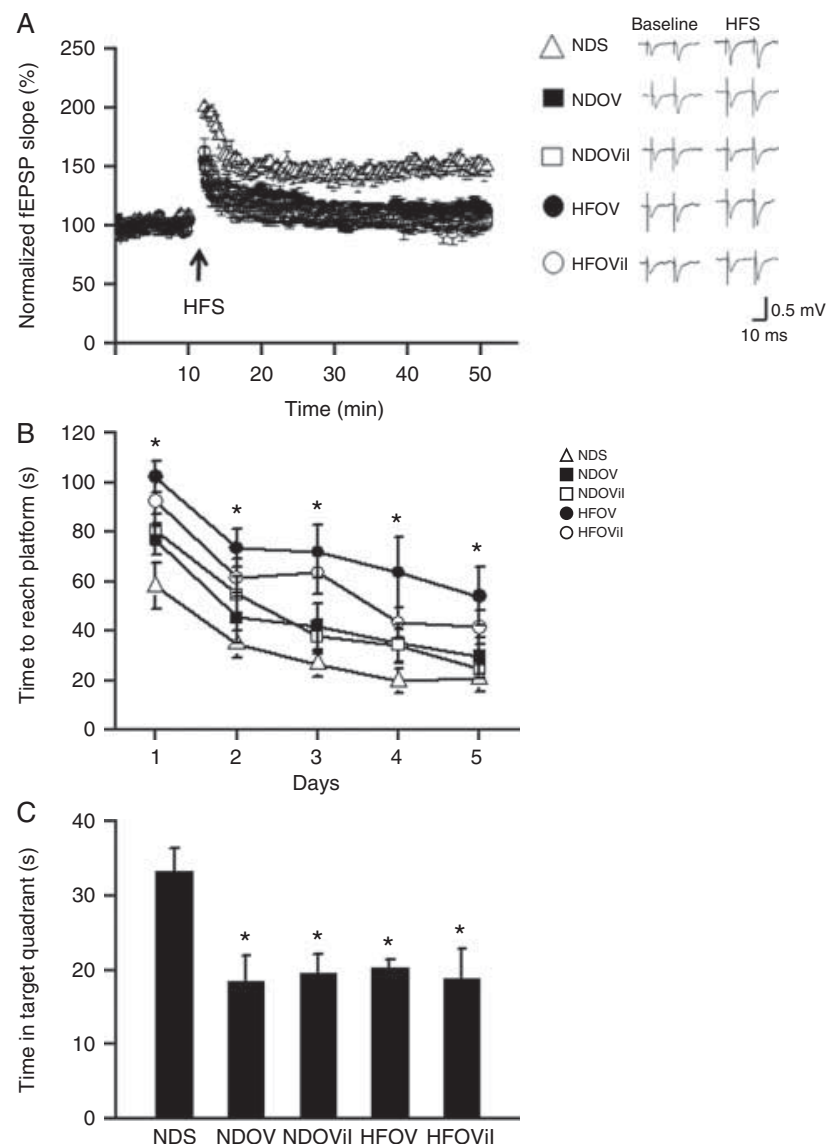

Figure 5

The effects of vildagliptin on hippocampal synaptic long-term potentiation (LTP) and the cognitive function determined by Morris water maze (MWM) test in testosterone-deprived rats with or without obesity. Both orchiectomized-operated groups (NDOV and HFOV rats) significantly reduced the percentage of normalized fEPSPs, when compared with that of NDS rats (A). The administration of vildagliptin for 4 weeks did not improve the percentage of normalized fEPSPs in both orchiectomized-operated groups (NDOVil and HFOVil), when compared with that of HFOV rats (A). HFOV rats and HFOVil rats significantly increased time to reach the platform in the acquisition test as well as had a decreased time spent in target quadrant in the probe test, when compared with that of NDS rats (B and C). NDOV rats had a significantly decreased time spent in the target quadrant in the probe test, but there was no changed in time to reach the platform in the acquisition test ( $\mathrm{B}$ and $\mathrm{C}$ ). The administration of vildagliptin for 4 weeks did not restore the time taken to reach the platform in HFOVil rats, and the time spent in the target quadrant in both NDOVil and HFOVil rats (B and C). NDS, sham-operated rats with normal diet feeding; NDOV, orchiectomizedoperated rats with normal diet feeding and treated with vehicle; NDOVil, orchiectomized-operated rats with normal diet feeding and treated with vildagliptin; HFOV, orchiectomized-operated rats with high-fat diet feeding and treated with vehicle; HFOVil, orchiectomized-operated rats with high-fat diet feeding and treated with vildagliptin; HFS, high frequency stimulation. ${ }^{*} P<0.05$ vs NDS.

depended on HFD-induced obesity, but not testosteronedeprivation, as confirmed by no change in insulin sensitivity in NDOV rats. Our findings are also consistent with a previous report by Christoffersen et al. (2006).
In addition, the present study demonstrated that ORX led to a loss of body weight. The reduction of daily food intake in the ORX group could be the explanation of weight loss in these rats. These findings are consistent with several previous studies, which showed that the reduction of body weight in ORX rats was caused by the reduction of daily food intake (Gentry \& Wade 1976, Borst \& Conover 2006) and muscle atrophy (Gao et al. 2005, Axell et al. 2006, Borst $\&$ Conover 2006). In contrast to our findings, the studies of Xia et al. (2013) found that ORX-induced testosterone deficiency increased the fasting blood glucose in mature adult (10 weeks old) Sprague-Dawley male rats. Jayaraman et al. (2014) demonstrated that ORX adult mice (3 months of age) had impaired insulin sensitivity. Since young adult male Wistar rats were used and were ORX at 5-6 weeks of age in the present study, the differences in species and age at the time of ORX procedure could be responsible for these inconsistent findings.

Interestingly, we found that HFOV rats, but not NDOV rats, developed brain insulin resistance as indicated by a reduction of insulin induced-LTD and decreased p-IR, as well as brain mitochondrial dysfunction through increased brain ROS production, brain mitochondrial depolarization and brain mitochondrial swelling. These findings and other previous studies indicated that obesity caused impaired brain mitochondrial function, resulting in brain insulin resistance (Pipatpiboon et al. 2012, 2013). Furthermore, our results demonstrated that testosterone deficiency alone had no effects on peripheral insulin resistance, brain insulin sensitivity as well as brain mitochondrial function. These findings suggest that testosterone deficiency is not required for the development of HFD-induced insulin resistance, and that factors contributing to peripheral and brain insulin resistance as well as brain mitochondrial dysfunction in this model could be mainly due to obesity.

Growing evidence demonstrated that testosterone deprivation is associated with cognitive decline in both men and animal models (Sakata et al. 2000, Hogervorst et al. 2001, 2004, Spritzer et al. 2011). We also found that testosterone-deprived rats impaired cognition and impaired hippocampal synaptic plasticity, but HFDinduced obesity did not aggravate those impairments in the testosterone-deprived condition. The present findings and other previous studies (Sakata et al. 2000, Naghdi et al. 2005, Sandstrom et al. 2006, Spritzer et al. 2011) indicated that testosterone plays an important role in cognitive function. Since brain mitochondrial function and brain insulin sensitivity were intact in NDO rats, these results indicate that they were not mainly contributed to

Published by Bioscientifica Ltd. 
impaired synaptic plasticity and cognitive decline in testosterone-deprived model.

In the present study, vildagliptin treatment improved peripheral insulin resistance, attenuated brain insulin resistance and partially improved brain mitochondrial dysfunction in testosterone-deprived obese rats. The possible underlying mechanisms of vildagliptin, which improve both peripheral and brain insulin sensitivity, could be due to its ability to i) inhibit the DPP4 activity, resulting in an increase in plasma active GLP1 levels, leading to improve insulin sensitivity and ii) act as an antioxidant agent. Although the level of GLP1 was not measured in the present study, our previous study already demonstrated that vildagliptin increased plasma and brain active GLP1 levels, leading to increased peripheral and brain insulin sensitivity in obese male rats (Burkey et al. 2005, Pipatpiboon et al. 2013, Gault et al. 2015). In addition, an anti-oxidant effect of vildagliptin was already demonstrated via reduced malondialdehyde (MDA) levels in the circulation and the brain in our previous report (Pintana et al. 2013). Although vildagliptin increased brain insulin sensitivity and decreased brain mitochondrial dysfunction, it could not restore hippocampal synaptic plasticity and cognitive function in testosterone-deprived rats with or without obesity. Furthermore, findings from previous studies (Sandstrom et al. 2006, Spritzer et al. 2011, McConnell et al. 2012) and the present study (Fig. 5B and C) suggest that testosterone plays a critical role in maintaining the normal function of cognition. These findings also indicate that the impairment of synaptic plasticity that leads to cognitive decline in testosterone-deprived model was not mainly influenced by brain insulin resistance.

\section{Conclusion}

Testosterone deprivation can lead to cognitive decline via impaired hippocampal synaptic plasticity. However, testosterone-deprived obese condition leads to not only the development of cognitive decline and impaired hippocampal synaptic plasticity, but also brain insulin resistance and brain mitochondrial dysfunction. These findings suggest that testosterone-deprivation with obese insulin-resistant condition causes severe brain dysfunction. Although DPP4 inhibitor, vildagliptin, improves peripheral and brain insulin sensitivity as well as decreases brain mitochondrial ROS production, it fails to restore hippocampal synaptic plasticity and cognitive function. Therefore, vildagliptin does not provide benefits on preventing cognitive decline in subjects under testosterone-deprived obese condition.

\section{Declaration of interest}

The authors declare that there is no conflict of interest that could be perceived as prejudicing the impartiality of the research reported.

\section{Funding}

This work was supported by grants from the Thailand Research Fund TRF-BRG5780016 (S C C), the Royal Golden Jubilee PhD program (PHD/0025/2555, H P and S C C), National Research Council of Thailand (S C C), a NSTDA Research Chair Grant from the National Science and Technology Development Agency (N C) and Chiang Mai University Excellent Center Award (N C).

\section{Author contribution statement}

H P performed the experiments, analyzed the data and wrote the manuscript. W P performed the experiments and analyzed the data. W P performed the experiments and analyzed the data. N C designed the study, analyzed the data and wrote the manuscript. S C C designed the study, performed the experiments, analyzed the data and wrote the manuscript.

\section{References}

Ahren B, Landin-Olsson M, Jansson PA, Svensson M, Holmes D \& Schweizer A 2004 Inhibition of dipeptidyl peptidase-4 reduces glycemia, sustains insulin levels, and reduces glucagon levels in type 2 diabetes. Journal of Clinical Endocrinology and Metabolism 89 2078-2084. (doi:10.1210/jc. 2003-031907)

Akiyama T, Tachibana I, Shirohara H, Watanabe N \& Otsuki M 1996 Highfat hypercaloric diet induces obesity, glucose intolerance and hyperlipidemia in normal adult male Wistar rat. Diabetes Research and Clinical Practice 31 27-35. (doi:10.1016/0168-8227(96)01205-3)

Arakawa H 2005 Age dependent effects of space limitation and social tension on open-field behavior in male rats. Physiology \& Behavior $\mathbf{8 4}$ 429-436. (doi:10.1016/j.physbeh.2005.01.008)

Axell AM, MacLean HE, Plant DR, Harcourt LJ, Davis JA, Jimenez M, Handelsman DJ, Lynch GS \& Zajac JD 2006 Continuous testosterone administration prevents skeletal muscle atrophy and enhances resistance to fatigue in orchidectomized male mice. American Journal of Physiology. Endocrinology and Metabolism 291 E506-E516. (doi:10.1152/ ajpendo.00058.2006)

Aydilek N \& Aksakal M 2005 Effects of testosterone on lipid peroxidation, lipid profiles and some coagulation parameters in rabbits. Journal of Veterinary Medicine. A, Physiology, Pathology, Clinical Medicine 52 436-439. (doi:10.1111/j.1439-0442.2005.00764.x)

Borst SE \& Conover CF 2006 Orchiectomized Fischer 344 male rat models body composition in hypogonadal state. Life Sciences 79 411-415. (doi:10.1016/j.lfs.2006.01.035)

Burkey BF, Li X, Bolognese L, Balkan B, Mone M, Russell M, Hughes TE \& Wang PR 2005 Acute and chronic effects of the incretin enhancer vildagliptin in insulin-resistant rats. Journal of Pharmacology and Experimental Therapeutics 315 688-695. (doi:10.1124/jpet.105.087064)

Christoffersen B, Raun K, Svendsen O, Fledelius C \& Golozoubova V 2006 Evalution of the castrated male Sprague-Dawley rat as a model of the metabolic syndrome and type 2 diabetes. International Journal of Obesity 30 1288-1297. (doi:10.1038/sj.ijo.0803261)

Christoffersen BO, Gade LP, Golozoubova V, Svendsen O \& Raun K 2010 Influence of castration-induced testosterone and estradiol deficiency on obesity and glucose metabolism in male Gottingen minipigs. Steroids 75 676-684. (doi:10.1016/j.steroids.2010.04.004)

Craft S 2005 Insulin resistance syndrome and Alzheimer's disease: age- and obesity-related effects on memory, amyloid, and inflammation.

Published by Bioscientifica Ltd. 
Neurobiology of Aging 26(Suppl 1) 65-69. (doi:10.1016/j.neurobiolaging. 2005.08.021)

Dulisch ML 1976 A castration procedure for the rabbit, rat, hamster, and guinea pig. Journal of Zoo Animal Medicine 7 8-11. (doi:10.2307/ 20094380)

Filova B, Ostatnikova D, Celec P \& Hodosy J 2013 The effect of testosterone on the formation of brain structures. Cells, Tissues, Organs 197 169-177. (doi:10.1159/000345567)

Gao W, Reiser PJ, Coss CC, Phelps MA, Kearbey JD, Miller DD \& Dalton JT 2005 Selective androgen receptor modulator treatment improves muscle strength and body composition and prevents bone loss in orchidectomized rats. Endocrinology 146 4887-4897. (doi:10.1210/en. 2005-0572)

Gault VA, Lennox R \& Flatt PR 2015 Sitagliptin, a dipeptidyl peptidase-4 inhibitor, improves recognition memory, oxidative stress and hippocampal neurogenesis and upregulates key genes involved in cognitive decline. Diabetes, Obesity \& Metabolism 17 403-413. (doi:10.1111/dom. 12432)

Gentry RT \& Wade GN 1976 Androgenic control of food intake and body weight in male rats. Journal of Comparative and Physiological Psychology 90 18-25. (doi:10.1037/h0077264)

Greenwood CE \& Winocur G 2005 High-fat diets, insulin resistance and declining cognitive function. Neurobiology of Aging 26(Suppl 1) 42-45. (doi:10.1016/j.neurobiolaging.2005.08.017)

Grossmann M, Thomas MC, Panagiotopoulos S, Sharpe K, Macisaac RJ, Clarke S, Zajac JD \& Jerums G 2008 Low testosterone levels are common and associated with insulin resistance in men with diabetes. Journal of Clinical Endocrinology and Metabolism 93 1834-1840. (doi:10.1210/jc. 2007-2177)

Haffner SM, Karhapaa P, Mykkanen L \& Laakso M 1994 Insulin resistance, body fat distribution, and sex hormones in men. Diabetes 43 212-219. (doi:10.2337/diab.43.2.212)

Hogervorst E, Williams J, Budge M, Barnetson L, Combrinck M \& Smith AD 2001 Serum total testosterone is lower in men with Alzheimer's disease. Neuro Endocrinology Letters 22 163-168.

Hogervorst E, Bandelow S, Combrinck M \& Smith AD 2004 Low free testosterone is an independent risk factor for Alzheimer's disease. Experimental Gerontology 39 1633-1639. (doi:10.1016/j.exger.2004. 06.019)

Janowsky JS 2006 Thinking with your gonads: testosterone and cognition. Trends in Cognitive Sciences 10 77-82. (doi:10.1016/j.tics.2005.12.010)

Jayaraman A, Lent-Schochet D \& Pike CJ 2014 Diet-induced obesity and low testosterone increase neuroinflammation and impair neural function. Journal of Neuroinflammation 11 162. (doi:10.1186/s12974014-0162-y)

Kahn BB \& Flier JS 2000 Obesity and insulin resistance. Journal of Clinical Investigation 106 473-481. (doi:10.1172/JCI10842)

McConnell SE, Alla J, Wheat E, Romeo RD, McEwen B \& Thornton JE 2012 The role of testicular hormones and luteinizing hormone in spatial memory in adult male rats. Hormones and Behavior 61 479-486. (doi:10.1016/j.yhbeh.2012.01.003)

Meydan S, Kus I, Tas U, Ogeturk M, Sancakdar E, Dabak DO, Zararsiz I \& Sarsilmaz M 2010 Effects of testosterone on orchiectomy-induced oxidative damage in the rat hippocampus. Journal of Chemical Neuroanatomy 40 281-285. (doi:10.1016/j.jchemneu.2010.07.006)

Naghdi N, Majlessi N \& Bozorgmehr T 2005 The effect of intrahippocampal injection of testosterone enanthate (an androgen receptor agonist) and anisomycin (protein synthesis inhibitor) on spatial learning and memory in adult, male rats. Behavioural Brain Research 156 263-268. (doi:10.1016/j.bbr.2004.05.032)

Pandolfi C, Pellegrini L, Sbalzarini G \& Mercantini F 1994 Obesity and insulin resistance. Minerva Medica 85 167-171.
Pintana H, Apaijai N, Pratchayasakul W, Chattipakorn N \& Chattipakorn SC 2012 Effects of metformin on learning and memory behaviors and brain mitochondrial functions in high fat diet induced insulin resistant rats. Life Sciences 91 409-414. (doi:10.1016/j.lfs.2012.08.017)

Pintana H, Apaijai N, Chattipakorn N \& Chattipakorn SC 2013 DPP-4 inhibitors improve cognition and brain mitochondrial function of insulin resistant rats. Journal of Endocrinology 218 1-11. (doi:10.1530/ JOE-12-0521)

Pipatpiboon N, Pratchayasakul W, Chattipakorn N \& Chattipakorn SC $2012 \operatorname{PPAR} \gamma$ agonist improves neuronal insulin receptor function in hippocampus and brain mitochondria function in rats with insulin resistance induced by long term high-fat diets. Endocrinology 153 329-338. (doi:10.1210/en.2011-1502)

Pipatpiboon N, Pintana H, Pratchayasakul W, Chattipakorn N \& Chattipakorn SC 2013 DPP4-inhibitor improves neuronal insulin receptor function, brain mitochondrial function and cognitive function in rats with insulin resistance induced by high-fat diet consumption. European Journal of Neuroscience 37 839-849. (doi:10.1111/ejn.12088)

Pratchayasakul W, Kerdphoo S, Petsophonsakul P, Pongchaidecha A, Chattipakorn N \& Chattipakorn SC 2011 Effects of high-fat diet on insulin receptor function in rat hippocampus and the level of neuronal corticosterone. Life Sciences 88 619-627. (doi:10.1016/ j.lfs.2011.02.003)

Sakata K, Tokue A \& Kawai N 2000 Altered synaptic transmission in the hippocampus of the castrated male mouse is reversed by testosterone replacement. Journal of Urology 163 1333-1338. (doi:10.1016/S00225347(05)67773-7)

Sandstrom NJ, Kim JH \& Wasserman MA 2006 Testosterone modulates performance on a spatial working memory task in male rats. Hormones and Behavior 50 18-26. (doi:10.1016/j.yhbeh.2005.09.008)

Shi Z, Araujo AB, Martin S, O’Loughlin P \& Wittert GA 2013 Longitudinal changes in testosterone over five years in community-dwelling men. Journal of Clinical Endocrinology and Metabolism 98 3289-3297. (doi:10.1210/jc.2012-3842)

Spritzer MD, Daviau ED, Coneeny MK, Engelman SM, Prince WT \& Rodriguez-Wisdom KN 2011 Effects of testosterone on spatial learning and memory in adult male rats. Hormones and Behavior 59 484-496. (doi:10.1016/j.yhbeh.2011.01.009)

Sripetchwandee J, Pipatpiboon N, Chattipakorn N \& Chattipakorn S 2014 Combined therapy of iron chelator and antioxidant completely restores brain dysfunction induced by iron toxicity. PLOS ONE 9 e85115. (doi:10.1371/journal.pone.0085115)

Stellato RK, Feldman HA, Hamdy O, Horton ES \& McKinlay JB 2000 Testosterone, sex hormone-binding globulin, and the development of type 2 diabetes in middle-aged men: prospective results from the Massachusetts male aging study. Diabetes Care 23 490-494. (doi:10.2337/diacare.23.4.490)

Stranahan AM, Norman ED, Lee K, Cutler RG, Telljohann RS, Egan JM \& Mattson MP 2008 Diet-induced insulin resistance impairs hippocampal synaptic plasticity and cognition in middle-aged rats. Hippocampus 18 1085-1088. (doi:10.1002/hipo.20470)

Vorhees CV \& Williams MT 2006 Morris water maze: procedures for assessing spatial and related forms of learning and memory. Nature Protocols 1 848-858. (doi:10.1038/nprot.2006.116)

Xia F, Xu X, Zhai H, Meng Y, Zhang H, Du S, Xu H, Wu H \& Lu Y 2013 Castration-induced testosterone deficiency increases fasting glucose associated with hepatic and extra-hepatic insulin resistance in adult male rats. Reproductive Biology and Endocrinology 11 106. (doi:10.1186/ 1477-7827-11-106)

Received in final form 17 May 2015

Accepted 26 May 2015

Accepted Preprint published online 27 May 2015 http://joe.endocrinology-journals.org

DOI: 10.1530/JOE-15-0099
(C) 2015 Society for Endocrinology Printed in Great Britain
Published by Bioscientifica Ltd 